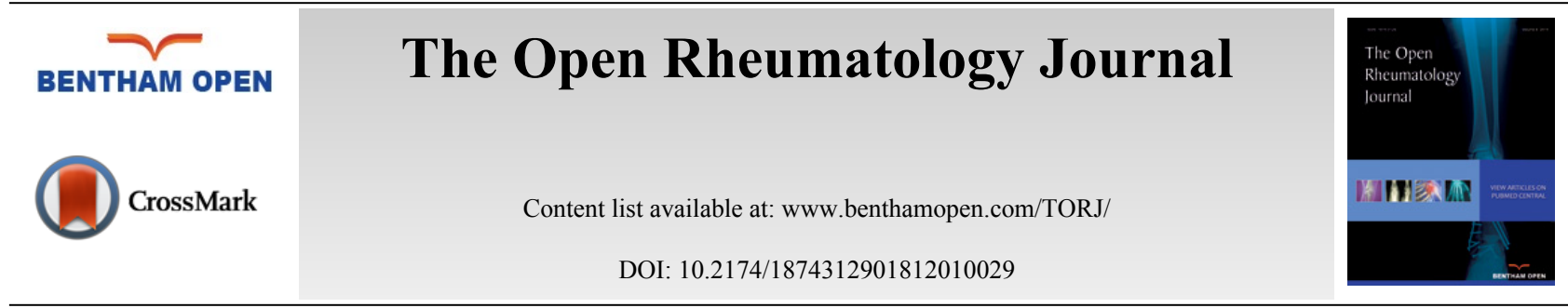

\title{
CORRIGENDUM
}

\section{Interethnic Variations and Clinical Features of Spondyloarthropathies in a Middle Eastern Country}

Mohammed Kamil Quraishi ${ }^{1, *}$, Humeira Badsha ${ }^{2}$, Bhavna Khan ${ }^{3}$, Muhammad Shahzeb ${ }^{4}$, Srilakshmi Hegde $^{5}$, Ayman Mofti ${ }^{5}$ and Kong Kok Ooi ${ }^{6}$

${ }^{I}$ Department of Urology, Medway Maritime Hospital, Kent, England, UK

${ }^{2}$ Department of Rheumatology. Dr. Humeira Badsha Medical Center, Dubai, UAE.

${ }^{3}$ Integrated Rheumatology and Arthritis Centre, Dubai Healthcare City, Dubai.

${ }^{4}$ Department of Medicine, Jinnah Medical College Hospital, Karachi, Pakistan.

${ }^{5}$ Department of Rheumatology, Al Biraa Arthritis \& Bone Center, Dubai, UAE.

${ }^{6}$ Department of Rheumatology, Tan Tock Seng Hospital, Singapore

Interethnic Variations and Clinical Features of Spondyloarthropathies in a Middle Eastern Country

The Open Rheumatology Journal, 2018, 12: 10-18

The correct address of corresponding author which is mentioned below:

Department of Urology, Medway Maritime Hospital, Windmill Road, Gillingham, Kent, UK ME7 5NY. Email: MKQURAISHI@doctors.org.uk

The original address provided was:

Department of Urology, Medway Maritime Hospital, 5 Miraj Avenue, Sparkhill, Birmingham, West Midlands, UK, B11 4JW; E-mail: MKQURAISHI@doctors.org.uk

The correct affiliation of Dr. Bhavna which is mentioned below:

Integrated Rheumatology and Arthritis Centre, Dubai Healthcare City, Dubai

The original affiliation provided was:

Department of Rheumatology, Dubai Mall Medical Center, Dubai, UAE

* Address correspondence to this author at the Department of Urology, Medway Maritime Hospital, Windmill Road, Gillingham, Kent, UK ME7 5NY; E-mail: MKQURAISHI@doctors.org.uk

(C) 2018 Quraishi et al.

This is an open access article distributed under the terms of the Creative Commons Attribution 4.0 International Public License (CC-BY 4.0), a copy of which is available at: (https://creativecommons.org/licenses/by/4.0/legalcode). This license permits unrestricted use, distribution, and reproduction in any medium, provided the original author and source are credited. 